\title{
Fadiga em Mulheres com Câncer de Mama Submetidas à Radioterapia
}

doi: https://doi.org/10.32635/2176-9745.RBC.2019v65n2.89

\author{
Fatigue in Women with Breast Cancer Submitted to Radiotherapy \\ Fatiga en Mujeres con Cáncer de Mama Sujeto a Radioterapia
}

Julyana Cândido Bahia'; Camila Mendonça Lima²; Murielly Marques de Oliveira³; Janaína Valadares Guimarães; Mônica de Oliveira Santos ${ }^{5}$; Dálete Delalibera Corrêa de Faria Mota ${ }^{6}$

Resumo

Introdução: A fadiga em mulheres com câncer de mama após a radioterapia é um dos efeitos colaterais mais debilitantes, sendo um sintoma subjetivo, multidimensional e multifatorial. Objetivo: Caracterizar a fadiga em pacientes com câncer de mama em radioterapia que realizam o tratamento no Serviço de Radioterapia de um hospital de referência em tratamento oncológico do Estado de Goiás. Método: Trata-se de um estudo longitudinal. A Escala de Fadiga de Piper - revisada foi utilizada para avaliação de fadiga no início (T1), meio (T2) e final (T3) da radioterapia. Resultados: A amostra foi composta por 89 mulheres. A prevalência de fadiga em T1 foi de $26,9 \%$. Houve aumento significativo da fadiga ao longo da radioterapia, sendo que, em T3, 50,8\% das mulheres apresentavam fadiga. Houve predomínio da fadiga moderada em T2 e T3, e o aumento mais significativo da intensidade da fadiga foi verificado do momento T1 para T2. A dimensão afetiva da fadiga apresentou escore mais alto comparado às dimensóes sensorial/psicológica. Conclusáo: A presença e a intensidade da fadiga durante a radioterapia aumentaram significativamente, predominando a fadiga moderada na última semana do tratamento. A magnitude da fadiga exibiu escores mais altos na dimensão afetiva nas avaliações. Portanto, atenção maior à fadiga durante a radioterapia precisa ser dada pelos profissionais de saúde.

Palavras-chave: Fadiga; Neoplasias da Mama/radioterapia; Radioterapia/efeitos adversos; Estudos Longitudinais; Mulheres.

\section{Abstract}

Introduction: Fatigue in women with breast cancer after radiotherapy is one of the most debilitating side effects, being a subjective, multidimensional, multifactorial symptom. Objective: To characterize fatigue in patients with breast cancer in radiotherapy who undergo treatment in the radiotherapy service of a reference hospital in cancer treatment in the State of Goiás. Method: This is a longitudinal study. The Piper Fatigue Scale - revised was used to evaluate fatigue at the beginning (T1), middle (T2) and final (T3) of the radiotherapy. Results: The sample consisted of 89 women. The prevalence of $\mathrm{T} 1$ fatigue was $26.9 \%$. There was a significant increase in fatigue during radiotherapy, and in T3, 50.8\% of the women presented fatigue. There was a predominance of moderate fatigue in $\mathrm{T} 2$ and $\mathrm{T} 3$, and the most significant increase in fatigue intensity was verified from $\mathrm{T} 1$ to T2. The affective dimension of fatigue presented a higher score compared to the sensorial/psychological dimensions. Conclusion: The presence and intensity of fatigue during radiotherapy increased significantly, with moderate fatigue predominating in the last week of treatment. The magnitude of fatigue exhibited higher scores in the affective dimension of the evaluations. Therefore, health professionals must focus more attention to fatigue during radiotherapy.

Key words: Fatigue; Breast Neoplasms/radiotherapy; Radiotherapy/adverse effects; Longitudinal Studies; Women.
Resumen

Introducción: La fatiga en mujeres con cáncer de mama después de la radioterapia es uno de los efectos colaterales más debilitantes, siendo un síntoma subjetivo, multidimensional, multifactorial. Objetivo: caracterizar la fatiga en pacientes con cáncer de mama en radioterapia que realizan el tratamiento en el servicio de radioterapia de un hospital de referencia en tratamiento oncológico del Estado de Goiás. Método: Se trata de un estudio longitudinal. La Escala de Fatiga de Piper - revisada fue utilizada para evaluación de fatiga al inicio (T1), medio (T2) y final (T3) de la radioterapia. Resultados: La muestra fue compuesta por 89 mujeres. La prevalencia de fatiga en T1 fue de 26,9\%. Se observó un aumento significativo de la fatiga a lo largo de la radioterapia siendo que, en T3, el 50,8\% de las mujeres presentaban fatiga. Se observó un predominio de la fatiga moderada en T2 y T3, y el aumento más significativo de la intensidad de la fatiga fue verificado del momento T1 para T2. La dimensión afectiva de la fatiga presentó una puntuación más alta en comparación con las dimensiones sensorial/psicológica. Conclusión: La presencia e intensidad de la fatiga durante la radioterapia aumentó significativamente, predominando la fatiga moderada en la última semana del tratamiento. La magnitud de la fatiga exhibió escores más altos en la dimensión afectiva en las evaluaciones. Por lo tanto, los profesionales de la salud deben prestar mayor atención a la fatiga durante la radioterapia.

Palabras clave: Fatiga; Neoplasias de la Mama/radioterapia; Radioterapia/ efectos adversos; Estudios Longitudinales; Mujeres.

\footnotetext{
${ }^{1}$ Universidade Federal de Goiás (UFG). Goiânia (GO), Brasil. Orcid iD: http://orcid.org/0000-0001-5027-6652

${ }^{2}$ Centro Brasileiro de Radioterapia, Oncologia e Mastologia (Cebrom). Goiânia (GO), Brasil. Orcid iD:http://orcid.org/0000-0001-5185-8363

${ }^{3}$ UFG. Goiânia (GO), Brasil. Orcid iD: http://orcid.org/0000-0002-9286-6893

${ }^{4}$ UFG. Goiânia (GO), Brasil. Orcid iD: http://orcid.org/0000-0003-1012-4405

${ }^{5}$ Instituto de Patologia Tropical e Saúde Pública (IPTSP). UFG. Goiânia (GO), Brasil. Orcid iD: http://orcid.org/0000-0001-7110-5430

${ }^{6}$ University of Wisconsin - Eau Claire. College of Nursing and Health Sciences. Eau Claire, Wisconsin (WI), Estados Unidos. Orcid iD: http://orcid.org/00000002-1323-4178

Endereço para correspondência: Julyana Cândido Bahia. Rua 227 - Setor Leste Universitário. Goiânia (GO), Brasil. CEP 74605-080. E-mail: julyanaweb@hotmail.com
} 


\section{INTRODUÇÃO}

De acordo com dados do Instituto Nacional de Câncer José Alencar Gomes da Silva (INCA), o câncer de mama é a primeira causa de morte por câncer em mulheres no Brasil e, para 2018, foram esperados 59.700 casos novos de câncer de mama ${ }^{1}$.

Entre os tratamentos utilizados na terapêutica do câncer de mama, está a radioterapia, ou teleterapia, um dos métodos mais indicados para o tratamento da doença ${ }^{2}$. No entanto, apesar de ser um tratamento eficaz, a radioterapia afeta a qualidade de vida e o equilíbrio corporal do indivíduo, desencadeando a fadiga, náusea, perda de apetite, perda de cabelo, depressão, ganho de peso, dificuldade respiratória, sono prejudicado e perda de força muscular ${ }^{3}$.

Fadiga em mulheres com câncer de mama após a radioterapia é um dos efeitos colaterais mais debilitantes, podendo afetar entre $84 \%$ a $86 \%$ das pacientes, sendo um sintoma subjetivo, multidimensional e multifatorial ${ }^{4}$. Pacientes fatigados expressam sentimentos de cansaço, incapacidade de manter a rotina usual, perda de libido, verbalização de uma constante falta de energia, entre outros ${ }^{5}$.

Desse modo, esse sintoma é muito frequente na última semana de radioterapia, sendo que, quanto mais intensa é a fadiga antes da radioterapia, maior será a intensidade desse sintoma durante o tratamento com a radiação ionizante. Além disso, é evidente que, em geral, a fadiga retorna ao nível pré-tratamento dois a três meses depois do término da radioterapia ${ }^{6}$.

A fadiga é um sintoma de difícil manejo, com fisiopatologia ainda não completamente compreendida, por isso controlar a evolução do quadro desde o início da doença é fator primordial e de competência da equipe interdisciplinar ${ }^{7}$. Assim, é imprescindível a caracterização desse sintoma em pacientes submetidos à radioterapia, uma vez que a fadiga é uma experiência que tem uma repercussão negativa significativa ${ }^{8}$.

Nesse sentido, a avaliação da fadiga em pacientes com câncer de mama em tratamento radioterápico convencional é essencial para o planejamento de intervenções individualizadas, como organização do ciclo atividade/repouso, orientação nutricional e manejo de possíveis fatores causais como depressão ou anemia ${ }^{8}$.

Diante disso, este estudo justifica-se na medida em que se faz necessário explorar os aspectos da fadiga em pacientes com câncer de mama submetidas à radioterapia em razão da lacuna de conhecimento que há sobre essa experiência multidimensional em estudos no Brasil e no mundo, bem como sobre suas características, dimensōes, intensidade e evolução, dificultando o planejamento de açóes mais efetivas para prevençáo e tratamento do sintoma por parte dos profissionais de saúde. Sabendo disso, desenvolveu-se o presente estudo, o qual buscou caracterizar a fadiga de mulheres com câncer de mama, submetidas à radioterapia convencional, quanto à presença, à intensidade e à magnitude nas dimensóes sensorial-psicológica, afetiva e comportamental a partir da Escala de Fadiga de Piper ${ }^{9,10}$.

\section{MÉTODO}

Trata-se de um estudo de coorte prospectiva, com seguimento em três momentos. A amostra foi constituída por 89 mulheres com diagnóstico de câncer de mama em tratamento radioterápico convencional. A coleta foi realizada no Serviço de Radioterapia de um hospital de referência em tratamento oncológico do Estado de Goiás, em atendimento à Resolução n. ${ }^{\circ}$ 466/2012 do Conselho Nacional de Saúde. O projeto do qual este estudo fez parte foi submetido à apreciação do Comitê de Ética em Pesquisa do hospital onde os dados foram coletados e aprovado sob Parecer n. ${ }^{\circ}$ 544.604, CAAE:14552713.3.0000.0031.

Os critérios de inclusão foram: ser do sexo feminino; apresentar câncer de mama, em qualquer estádio; idade igual ou superior a 18 anos; indicaçáo de 20 ou mais fraçôes de radioterapia, com dose de, no mínimo, 180 cGy por dia; capacidade de comunicaçấo e compreensão preservadas. Os critérios de exclusão foram: quimioterapia concomitante à radioterapia; suspensão da radioterapia por 15 dias ou mais, ou por três ou mais suspensôes, independentemente do número de dias.

As participantes da pesquisa foram recrutadas entre julho de 2015 e abril de 2016 durante visitas realizadas por enfermeiros e acadêmicos de enfermagem treinados. As visitas eram realizadas durante três dias da semana no setor de radioterapia do hospital e aquelas pacientes que atendiam aos critérios de inclusão eram convidadas a participar da pesquisa. Assim, aquelas que aceitaram participar do estudo após leitura, esclarecimento de dúvidas e assinatura do Termo de Consentimento Livre e Esclarecido (TCLE), em duas vias, eram entrevistadas durante o período de espera para a seçáo de radioterapia, em um consultório reservado para coleta dos dados. Desse modo, as informaçôes da pesquisa foram compostas de dados provenientes de entrevista e consulta ao prontuário. A coleta de dados foi realizada em três momentos: o primeiro momento, desde a simulação até a quarta ou quinta sessão de radioterapia (T1); o segundo momento, 15 dias após o início da radioterapia \pm dois dias (T2); e o terceiro momento, na última semana do tratamento (T3).

A Escala de Fadiga de Piper - revisada, validada para uso no Brasil em 2009, foi utilizada para avaliação 
de fadiga ${ }^{10}$. É um instrumento multidimensional de autorrelato, composto por 22 itens fechados e quatro itens abertos. Os 22 itens compreendem três dimensóes de fadiga: comportamental, afetiva e sensorial-psicológica. Cada item é apresentado em escala numérica (0 a 10) e o escore total é calculado pela média de todos os itens do instrumento, e os escores das dimensôes são calculados a partir da média dos itens contidos em cada dimensão, sendo que os mais altos indicam maior fadiga $^{9,10}$. As pacientes foram consideradas fatigadas quando apresentaram escore diferente de zero na Escala de Piper - revisada, seguindo a sugestáo do consenso National comprehensive Cancer Network para classificação da fadiga, que estabeleceu o ponto de corte no escore 4 para discriminar indivíduos fatigados dos não fatigados. Além disso, para classificação da intensidade da fadiga com base no escore total, constituíram-se três níveis: leve (escore maior que 0 e menor que 4); moderada (escore igual ou maior que 4 e menor que 6); e intensa (escore igual ou maior que 6 até 10$)^{11}$.

Além de dados sobre fadiga, a coleta incluiu dados sociodemográficos, clínicos, relacionados ao câncer e seus tratamentos por meio de um instrumento desenvolvido pelas pesquisadoras envolvidas no projeto de pesquisa. Tais dados compuseram a caracterização da amostra em estudo.

As informaçôes obtidas foram tabuladas na planilha do Microsoft Excel ${ }^{\circledR}$ e os resultados, apresentados na forma de tabelas, gráficos e/ou quadros, descritos em números absolutos e percentuais. A análise dos dados ocorreu por meio do programa estatístico Statistical Package for Social Science (SPSS) e, para todos os testes, considerou-se um nível de significância de 5\%. Os escores obtidos na Escala de Fadiga de Piper - revisada seguiram distribuição normal nos três momentos (T1, T2 e T3). Testes paramétricos avaliaram a diferença entre as médias das dimensôes de fadiga e do escore total de fadiga em um mesmo momento de avaliação (teste t para uma amostra) e, para avaliação da diferença entre as médias de um mesmo domínio e o escore total ao longo dos três momentos de avaliação, a análise de variância (Anova) para medidas repetidas.

\section{RESULTADOS}

Durante o período de estudo, 89 mulheres foram incluídas na coorte (T1); destas, 86 pacientes foram avaliadas em T2 e $81 \mathrm{em} \mathrm{T3,} \mathrm{com} \mathrm{um} \mathrm{total} \mathrm{de} \mathrm{oito} \mathrm{perdas}$ ao longo das três avaliaçóes; entre elas, uma perda por motivo de recusa da paciente em participar das avaliaçóes subsequentes, uma em virtude de suspensão por mais de 15 dias e quatro por impossibilidade de realizar a entrevista no momento oportuno por parte dos pesquisadores. Foram excluídas do estudo pacientes que iniciaram quimioterapia concomitante à radioterapia, ao longo das avaliaçóes, constituindo duas participantes.

De modo geral, as pacientes do estudo receberam doses fracionadas de radioterapia que variaram de 180 a 267 cGy e todas foram submetidas à cirurgia antes da radioterapia. Além disso, conforme o estadiamento dos tumores pela classificação dos tumores malignos, 43 (48,3\%) mulheres estavam em estádio III, 37 (41,6\%) em estádio II e nove $(10,1 \%)$ em estádio $\mathrm{I}^{12}$.

Conforme apresentado na Tabela 1, a amostra foi composta por 89 pacientes, com média de idade de 53,9 anos; até seis anos de estudo (53,4\%); predomínio de renda per capita menor que um salário mínimo (67,8\%); a maioria $(60,2 \%)$ tinha companheiro fixo e náo era fumante $(92,1 \%)$.

A quimioterapia neoadjuvante foi administrada em $39,3 \%$ das pacientes e a quimioterapia adjuvante em 49,4\% da amostra; $61,3 \%$ das pacientes apresentaram hemoglobina $\leq 12,4 \mathrm{~g} / \mathrm{dl}$. A interrupção do tratamento foi verificada em 30 mulheres $(34,5 \%)$. Destas 20 $(66,6 \%)$, com interrupção menor ou igual a três dias, seis $(20,0 \%)$ com interrupção por quatro a oito dias, e outras quatro $(13,3 \%)$ com interrupção por nove a 14 dias. $\mathrm{O}$ motivo das interrupçóes esteve relacionado a problemas decorrentes da viabilidade do transporte até o hospital, conforme relato das pacientes, suspensão temporária da atividade dos equipamentos de radioterapia por defeito e manutenção, e por complicaçóes de pele (radiodermite) decorrentes da radioterapia (Tabela 1).

Conforme a Tabela 2 , a prevalência da fadiga no início do tratamento estava pesente em $26,9 \%$ das pacientes, sendo que foi identificada a ocorrência de novos casos de fadiga ao longo da radioterapia. No que se refere à intensidade da fadiga, em T1 tem-se igual número de mulheres com fadiga leve e fadiga moderada. Já em T2 e T3, foi identificado predomínio da fadiga moderada entre as pacientes do estudo, com $20,9 \%$ e $32,1 \%$, respectivamente. No geral, com base no escore total, um maior número de mulheres, $32,1 \%$, apresentou nível moderado de fadiga ao final do tratamento com escore médio de 6,0 (DP=1,7).

A intensidade média de fadiga para cada dimensão da Escala de Fadiga de Piper e em cada momento do estudo está apresentada na Tabela 3. Evidenciou-se um aumento gradativo da intensidade média de fadiga nos três momentos do estudo (T1, T2 e T3), tanto observando o escore total como o escore de cada dimensão. $\mathrm{O}$ teste Anova para medidas repetidas foi conduzido para avaliar a hipótese nula de que náo houve mudança nos escores de fadiga total e por dimensão ao longo da radioterapia. Os resultados indicam significativo efeito ao longo da terapia (Wilks Lambda $=0,821, \mathrm{p}<0,001$ ). Portanto, há 
Tabela 1. Distribuição das mulheres submetidas à radioterapia segundo as características clínicas e sociodemográficas. Goiânia, GO, Brasil, 2015 ( $n=89)$

\begin{tabular}{|c|c|c|}
\hline \multicolumn{3}{|l|}{ Características } \\
\hline Idade: & \multirow{2}{*}{\multicolumn{2}{|c|}{$\begin{array}{c}53,9 \text { anos }(14,9) ; \\
31,0-83,0\end{array}$}} \\
\hline \multirow[t]{2}{*}{ Média (DP); min.-máx. } & & \\
\hline & $\mathbf{n}$ & $\%$ \\
\hline \multicolumn{3}{|l|}{ Escolaridade (anos) } \\
\hline $0-7$ & 47 & 53,4 \\
\hline $7-20$ & 41 & 46,6 \\
\hline \multicolumn{3}{|l|}{ Renda per capita } \\
\hline$<1 S M$ & 59 & 67,8 \\
\hline $2-4 \mathrm{SM}$ & 28 & 32,2 \\
\hline \multicolumn{3}{|l|}{ Com companheiro } \\
\hline Sim & 53 & 60,2 \\
\hline Não & 35 & 39,8 \\
\hline \multicolumn{3}{|l|}{ Cor da pele } \\
\hline Branco & 34 & 38,6 \\
\hline Pardo & 42 & 47,7 \\
\hline Negro & 7 & 8,0 \\
\hline Amarelo & 5 & 5,7 \\
\hline Indígena & 0 & 0,0 \\
\hline \multicolumn{3}{|l|}{ Tabagismo } \\
\hline Fumante & 82 & 92,1 \\
\hline Não fumante & 07 & 7,9 \\
\hline \multicolumn{3}{|c|}{ Quimioterapia neoadjuvante } \\
\hline Sim & 35 & 39,3 \\
\hline Não & 54 & 60,7 \\
\hline \multicolumn{3}{|l|}{ Quimioterapia adjuvante } \\
\hline Sim & 44 & 49,4 \\
\hline Não & 45 & 50,6 \\
\hline \multicolumn{3}{|l|}{$\mathrm{IMC}\left(\mathrm{Kg} / \mathrm{m}^{2}\right)$} \\
\hline$\leq 25$ & 37 & 41,6 \\
\hline$>25$ & 52 & 58,4 \\
\hline \multicolumn{3}{|l|}{ Hormonioterapia } \\
\hline Sim & 30 & 34,5 \\
\hline Não & 57 & 65,5 \\
\hline \multicolumn{3}{|l|}{ Hemoglobina (g/dl) } \\
\hline$\geq 8$ e $<10$ & 2 & 3,2 \\
\hline$\geq 10$ e $\leq 12,4$ & 36 & 58,1 \\
\hline$>12,5$ & 24 & 38,7 \\
\hline \multicolumn{3}{|c|}{ Interrupção do tratamento } \\
\hline Não interrompeu & 57 & 65,5 \\
\hline Interrompeu & 30 & 34,5 \\
\hline \multicolumn{3}{|l|}{ Comorbidade } \\
\hline Presença de $\geq 1$ & 75 & 84,3 \\
\hline Ausência & 14 & 15,7 \\
\hline
\end{tabular}

Legendas: $\mathrm{SM}=$ Salários mínimos; $\mathrm{DP}=$ Desvio-padrão; $\mathrm{IMC}=$ Índice de massa corpórea.
Tabela 2. Classificação de fadiga segundo as categorias ordinais pela Escala de Piper na $1^{a}$ avaliação (T1), $2^{a}$ avaliação (T2) e $3^{a}$ avaliação (T3). Goiânia, GO, Brasil, $2015(\mathrm{n}=89)$

\begin{tabular}{ccccc}
\hline $\begin{array}{c}\text { Presença } \\
\text { da } \\
\text { fadiga }\end{array}$ & \multicolumn{3}{|c}{$\begin{array}{c}\text { Classificação da fadiga } \\
\mathbf{n}(\%)\end{array}$} \\
\hline & $\mathbf{n}(\%)$ & Leve & Moderada & Intensa \\
$\mathrm{T} 1(\mathrm{n}=89)$ & 24 & 10 & 10 & 4 \\
& $(26,9)$ & $(11,2)$ & $(11,2)$ & $(4,5)$ \\
$\mathrm{T} 2(\mathrm{n}=86)$ & 35 & 5 & 18 & 12 \\
& $(40,7)$ & $(5,8)$ & $(20,9)$ & $(14,0)$ \\
$\mathrm{T} 3(\mathrm{n}=81)$ & 41 & 3 & 26 & 12 \\
& $(50,6)$ & $(3,7)$ & $(32,1)$ & $(14,8)$ \\
\hline
\end{tabular}

Tabela 3. Classificação de fadiga segundo intensidade de modo contínuo e dimensões pela Escala de Piper na la avaliação (T1), 2 a avaliação (T2) e 3a avaliação (T3). Goiânia, GO, Brasil, 2015 (n=89)

\begin{tabular}{lccc}
\hline & \multicolumn{3}{c}{$\begin{array}{l}\text { Intensidade média da fadiga } \\
\text { (desvio-padrão) }\end{array}$} \\
\hline & Comportamentalt & Afetivat & $\begin{array}{c}\text { Sensorial/ } \\
\text { Psicológicat }\end{array}$ \\
T1 $^{*}$ & $5,0(2,2)$ & $6,9(2,4)$ & $5,1(2,6)$ \\
T2 $^{*}$ & $5,7(2,2)$ & $7,5(2,5)$ & $5,6(2,0)$ \\
T3* $^{*}$ & $6,1(2,0)$ & $8,2(2,3)$ & $5,7(1,9)$ \\
\hline Total & $5,2(2,1)$ & $6,0(1,9)$ & $6,4(1,8)$ \\
\hline
\end{tabular}

Legendas: *Teste Anova realizado em cada momento da avaliaçáo T1, T2 e T3; $\dagger$ Teste t realizado em cada dimensão.

evidência para rejeitar a hipótese nula. Comparaçôes por pares indicaram que a diferença foi significativa $(\mathrm{p}=0,001$ e $\mathrm{p}<0,001$, respectivamente) entre fadiga total em $\mathrm{T} 1 \mathrm{e}$ $\mathrm{T} 2$ em T1 e T3. A diferença entre os escores da dimensão comportamental de fadiga foi significativa entre T1 e T2 $(p=0,015)$ e entre T1 eT3 $(p=0,001)$. A diferença entre os escores da dimensão afetiva de fadiga foi significativa entre T1 e T2 ( $\mathrm{p}=0,003)$ e entre T1 e T3 ( $<<0,001)$. Diferenças significativas também foram observadas entre os escores $\mathrm{da}$ dimensão sensorial/psicológica, entre T1 e T2 ( $\mathrm{p}=0,003)$, entre T1 e T3 $(p<0,001)$ e entre T2 e T3 $(p<0,001)$.

Quanto à magnitude desse sintoma nas dimensôes avaliadas pela escala, as médias dos escores da dimensão afetiva de fadiga foram significativamente maiores $(\mathrm{p}=0,02)$ do que a média dos escores de cada uma das dimensôes comportamental, sensorial/pscológica e do escore total em T1, T2 e T3 (Tabela 3). Em T3, também houve diferença significativa entre o escore da dimensão sensorial/psicológica e o escore total de fadiga. Não houve diferença significativa entre as demais dimensôes da Escala de Piper e o escore total.

Entre as mulheres que referiram fadiga, parte respondeu às questóes abertas da Piper; em T1, 10 de 24, em T2, 22 de 35 e, em T3, 15 de 41 responderam. As 
pacientes fatigadas em $\mathrm{T} 1$ referiram que experimentavam fadiga fazia sete meses ( $\mathrm{DP}=3,8$; mediana $=7)$, em média.

Segundo as participantes fatigadas em T1, a quimioterapia foi considerada a causa mais comum da fadiga, seguida de fatores emocionais (estresse, medo, nervosismo) e o próprio câncer. Em T2, a quimioterapia foi citada como causa de fadiga, mas a radioterapia foi a mais comumente mencionada, seguida do câncer, problemas relacionados à radioterapia (deslocamento diário, duração do tratamento, tempo de espera, ambiente hospitalar, moradia temporária, por exemplo), problemas pessoais (desemprego, problemas financeiros, filhos etc.) e fatores emocionais (estresse, preocupação, ansiedade). Em $\mathrm{T} 3$, todas as respondentes referiram a radioterapia como sendo uma das causas de sua fadiga. Além disso, também foram referidos o tratamento quimioterápico prévio e os fatores emocionais e pessoais citados previamente.

Atividades de distração como passear, socializar com familiares e amigos, assistir televisão, jogar no celular ou computador foram aquelas mais adotadas entre as participantes fatigadas em T1 para alívio de fadiga, seguidas de descansar e dormir. Atividades religiosa e física foram mencionadas por uma mulher. Em T2, a ação mais citada para aliviar fadiga foi descansar e dormir; outras ações citadas em menor número foram atividades de distração, atividade física, atividade religiosa e trabalhar. Semelhante ao momento T2, em T3, a maioria das mulheres referiu descansar e dormir como as açóes mais adotadas para aliviar fadiga. Além das demais atividades de distração, atividade física e religiosa, mencionadas em menor número, houve um caso de menção em terapias alternativas (uso de ervas).

Em todas as três avaliaçóes, sonolência foi a palavra comumente citada para caracterização da sensaçáo de fadiga. Quando questionadas sobre a presença de outros sintomas, em T1, os sintomas mais citados foram dor $(\mathrm{n}=40,44,9 \%) \mathrm{e}$ prejuízo de sono ( $\mathrm{n}=21,23,6 \%)$. Em T2, dor foi referida por 38 (44,2\%) mulheres e prejuízo do sono por 28 (32,6\%) mulheres. Em T3, o relato de dor e prejuízo de sono foi de $39(48,1 \%)$ e $31(38,3 \%)$, respectivamente.

\section{DISCUSSÃO}

Este estudo avaliou a fadiga em três momentos, desde a simulação da radioterapia até a quarta ou a quinta sessão de radioterapia (T1), quatro semanas após o início da radioterapia \pm dois dias (T2) e na última semana do tratamento (T3). Ao descrever os dados detalhados sobre a evolução da fadiga durante o tratamento radioterápico, o presente estudo traz dados que contribuem com evidências sólidas sobre fadiga em mulheres com câncer de mama submetidas à radioterapia convencional.
Os estudos sobre a fadiga relacionada ao câncer apontam que é um sintoma comum e tratável que interfere significativamente em diversos aspectos da vida das pacientes com câncer de mama e está relacionada a múltiplos fatores incluindo o tratamento com radioterapia. Sendo assim, pacientes reportam a fadiga como um dos sintomas mais importantes e estressantes relacionados ao câncer e seu tratamento ${ }^{13-15}$. No entanto, apesar de o aumento dos níveis de fadiga resultante de tratamentos para o câncer ainda não ser consenso na literatura, haja vista seu caráter multidimensional e multifatorial, estudos relatam aumento da presença e da intensidade desse sintoma ao longo da radioterapia, sendo, portanto, um fator independente capaz de interferir na qualidade de vida e nos aspectos cognitivos-comportamentais ${ }^{13,15-18}$.

No presente estudo, a fadiga esteve presente em 24 pacientes $(26,9 \%)$ desde o início da radioterapia (T1), mesmo em mulheres que não haviam iniciado a radioterapia, sendo que, na terceira avaliação, mais da metade das mulheres apresentavam esse sintoma $(50,8 \%)$. Esse fato é preocupante na medida em que o nível de fadiga pré-tratamento pode ser um importante fator de risco para fadiga crônica e diminuição da qualidade de vida como encontrado em estudo realizado na Alemanha em que se avaliou os níveis de fadiga pré e pós-radioterapia ${ }^{19}$.

Em investigaçóes que utilizaram o mesmo ponto de corte $(\geq 4)$ na avaliação desse sintoma, verificou-se variação da presença da fadiga. Em estudo realizado na cidade de São Paulo, 31,9\% da amostra de 163 pacientes com câncer de mama apresentaram fadiga clinicamente relevante ${ }^{20}$.

Tendo em vista que muitas pacientes deste estudo iniciaram o tratamento apresentando algum grau de fadiga, a partir da classificação da intensidade da fadiga (leve, moderada, intensa), no último momento da avaliação, quase a metade $(47 \%)$ apresentou fadiga moderada e intensa (Tabela 2); já no estudo de Lamino et al. ${ }^{20}$, os autores encontraram predomínio de fadiga moderada e intensa em 64,2\% das pacientes.

Em outro estudo, realizado na Grécia, com 106 pacientes com câncer de mama em radioterapia, 13\% das pacientes apresentaram níveis moderado a intenso de fadiga no início da radioterapia ${ }^{15}$. Portanto, tais resultados indicam a importância de identificar esses pacientes em estágio inicial para que intervençôes apropriadas possam ser implementadas.

A intensidade média da fadiga ao final da radioterapia nas mulheres deste estudo foi de 6,0 ( $\mathrm{DP}=1,7)$, o que indica fadiga moderada (Tabela 3). Além disso, o aumento mais significativo da presença de fadiga de intensidades moderada e intensa $(\mathrm{p}<0,05)$ foi verificado do momento T1 para T2, com aumentos de $86,6 \%$ e $211,1 \%$, respectivamente (Tabela 2 ). 
Com relação à magnitude desse sintoma, a fadiga afetiva predominou quando comparada às outras dimensões avaliadas. Sabendo que essa dimensão busca identificar o significado ou a interpretação atribuída à fadiga, esse resultado indica o quanto ela interfere negativamente na vida das pacientes em virtude do seu caráter destruidor, inaceitável e desagradável ${ }^{10}$. Portanto, profissionais devem incluir intervençóes que envolvam a dimensão afetiva da pessoa, desenvolvendo a autoeficácia das mulheres e mostrando que a fadiga é um sintoma que pode ser controlado, mesmo que não curado.

Considerando a realidade vivida no local de coleta de dados e ao trocar experiência com colegas de outros serviços de radioterapia no Brasil, é notória a necessidade de capacitar os profissionais da área para diagnóstico e manejo de fadiga. No presente estudo, é possível presumir que esse sintoma se agravou desde o início da radioterapia sem que houvesse intervençấo por parte dos profissionais para sua redução e alívio, desse modo, é importante destacar que a fadiga pode limitar ou impedir a continuidade do tratamento ${ }^{16,17,20-22}$. Mais grave ainda, a partir das açóes adotadas pelas mulheres para alívio de fadiga, a maioria optou por descansar ou dormir, o que é o menos recomendado para o controle de fadiga.

Por fim, reconhece-se que a fadiga é um dos problemas da mulher com câncer de mama em radioterapia. Outros sintomas comumente reportados, dor e prejuízo de sono, podem se apresentar em um agrupamento (cluster) de sintomas e influenciar a intensidade da fadiga. Dessa forma, profissionais devem ser ativos e proativos na prevenção, identificação e controle das respostas humanas frente ao câncer e o tratamento empregado.

A presente pesquisa contribui para aumentar a compreensão sobre a fadiga e a radioterapia como fator independente de causa desse sintoma. Desse modo, o tratamento oncológico em mulheres com câncer de mama demanda extrema capacidade de adaptaçáo às novas condiçóes advindas desse processo. E, apesar da limitada assistência à fadiga, as mulheres reconhecem por si só a radioterapia como sendo uma causa importante do sintoma. Diante disso, esses resultados representam motivo de preocupaçáo por se tratar de um sintoma caracterizado como clinicamente relevante nessas pacientes com câncer de mama, em especial, naquelas mulheres que apresentavam fadiga intensa ao final do tratamento ${ }^{12,17,20,22}$.

\section{CONCLUSÃO}

A presença e a intensidade da fadiga durante a radioterapia aumentaram significativamente, predominando a fadiga moderada na última semana do tratamento. Além disso, com a caracterização da fadiga quanto à magnitude, pelas dimensóes da Escala de Fadiga de Piper - revisada, identificou-se que a dimensão afetiva prevaleceu com escores mais altos em todas as avaliaçóes demonstrando a percepção negativa das pacientes com relaçáo a esse sintoma, sendo referido de forma destruidora, anormal, desagradável na sua vida.

Esses resultados contribuem para o crescente corpo de evidências sobre a fadiga induzida pela radiação e fornecem base importante para que se caminhe em direçáo a intervençóes de enfermagem efetivas e apropriadas, bem como da equipe multiprofissional envolvida no cuidado a essas mulheres. Assim, este estudo reflete a necessidade de maior atenção à fadiga durante a radioterapia por parte dos profissionais da saúde com a utilização de escalas adequadas para detecção precoce e alívio desse sintoma que acarreta sofrimento e prejuízos à qualidade de vida das pacientes.

\section{CONTRIBUIÇÕES}

Todos os autores contribuíram substancialmente na concepção e no planejamento do estudo; na obtenção, na análise e/ou interpretação dos dados; assim como na redação e revisão crítica e aprovação final da versão publicada.

\section{DECLARAÇÃO DE CONFLITO DE INTERESSES}

Nada a declarar.

\section{FONTES DE FINANCIAMENTO}

Não há.

\section{REFERÊNCIAS}

1. Instituto Nacional de Câncer Jose de Alencar Gomes da Silva. Tipos de câncer: câncer de mama [Internet]. Rio de Janeiro: INCA; [data desconhecida] - [modificado 2019 ago. 27; acesso 2018 jan. 17]. Disponivel em: https:// www.inca.gov.br/tipos-de-cancer/cancer-de-mama

2. Instituto Nacional de Câncer Jose de Alencar Gomes da Silva. Como é feita a radioterapia? [Internet]. Rio de Janeiro: INCA; [data desconhecida] - [acesso 2019 out. 11]. Disponível em: https://www.inca.gov.br/perguntasfrequentes/como-e-feita-radioterapia

3. Loyola EAC, Borges ML, Magalhães PAP, et al. Rehabilitation group: benefits and barriers in the perspective of women with breast cancer. Texto Context Enferm. 2017;26(1):e3250015. doi: http://dx.doi. org/10.1590/0104-07072017003250015

4. Banipal RPS, Singh H, Singh B. Assessment of cancerrelated fatigue among cancer patients receiving various therapies: a cross-sectional observational study. Indian 
J Palliat Care. 2017;23(2):207-211. doi: http://dx.doi. org/10.4103/IJPC.IJPC_135_16

5. Oliveira TSG, Neris RR, Santos LNT, et al. Perfil de mulheres com câncer de mama tratadas com quimioterapia. Rev Enferm UFPE [Internet]. 2016 [acesso 2018 mar.10];10(11):4097-103. Disponível em: https://periodicos.ufpe.br/revistas/revistaenfermagem/ article/download/11496/13361

6. Ishikawa NM, Derchain SFM, Thuler LCS. Fadiga em pacientes com câncer de mama em tratamento adjuvante. Rev Bras Cancerol [Internet]. 2005 [acesso 2018 maio 9];51(4):313-318. Disponível em: https://rbc.inca.gov. br/index2.php

7. Mota DDCF, Pimenta CAM. Fadiga em pacientes com câncer avançado: conceito, avaliaçấo e intervenção. Rev Bras Cancer [Internet]. 2002 [acesso $2016 \mathrm{fev}$. 7];48(4):577-83. Disponível em: https://rbc.inca.gov. br/index2.php

8. Oliveira MM, Oliveira GF, Souza-Talarico JN, et al. Surgical oncology: evolution of postoperative fatigue and factors related to its severity. Clin J Oncol Nurs. 2016;20(1):E3-8. doi: http://dx.doi.org/10.1188/16. CJON.E3-E8

9. Piper B, Lindsey A, Dodd M, et al. The development of an instrument to measure the subjective dimension of fatigue. In: Funk SG, Tournquist PM, Campagne MT, editors. Key aspects of comfort: management of pain, fatigue, and nausea. New York: Springer Pub. Co.; c1989. p. 199-207.

10. Mota DDCF, Pimenta CAM, Piper BF. Fatigue in Brazilian cancer patients, caregivers, and nursing students: a psychometric validation study of the piper fatigue scalerevised. Support Care Cancer. 2009;17(6):645-52. doi: http://dx.doi.org/10.1007/s00520-008-0518-x

11. National Comprehensive Cancer Network (NCCN). Clinical practice guidelines in oncology: cancer-related fatigue, Version 1 [Internet]. 2015 [cited 2018 Oct 12]. Available from: https:/www.nccn.org/professionals/ physician_gls/pdf/fatigue.pdf

12. Instituto Nacional de Câncer. TNM: classificação de tumores malignos [TNM: classification of malignant tumours] [Internet]. 6.ed. Rio de Janeiro: INCA; 2004 [acesso 2018 maio 9]. Disponível em: http://bvsms. saude.gov.br/bvs/publicacoes/inca/tnm2.pdf

13. Alcântara-Silva TRM, Freitas-Junior R, Freitas NMA, et al. Fatigue related to radiotherapy for breast and/or gynaecological cancer: a systematic review. J Clin Nurs. 2013;22(19-20):2679-86. doi: https://doi.org/10.1111/ jocn. 12236

14. Kangas M, Bovbjerg DH, Montgomery GH. Cancerrelated fatigue: a systematic and meta-analytic review of non-pharmacological therapies for cancer patients. Psychol Bull. 2008;134(5):700-741. doi: https://doi. org/10.1037/a0012825
15. Lavdaniti M, Patiraki E, Dafni U, et al. Prospective Assessment of Fatigue and Health status in Greek patients with breast cancer undergoing adjuvant radiotherapy. Oncol Nurs Forum. 2006;33(3):603-610. doi: https:// doi.org/10.1188/06.ONF.603-610

16. Sucala M, Schnur JB, Brackman EH, et al. The role of specific and core dysfunctional beliefs in breast cancer radiotherapy patients' fatigue. J Health Psychol. 2014;19(8):957-65. doi: https://doi. org/10.1177/1359105313482166

17. Canário ACG, Cabral PUL, Paiva LC, et al. Physical activity, fatigue and quality of life in breast cancer patients. Rev Assoc Med Bras. 2016;62(1):38-44. doi: http://dx.doi.org/10.1590/1806-9282.62.01.38

18. Fortunato LA, Santos CM, Morales AP, et al. Pacientes portadoras de câncer de mama, submetidas à radioterapia com acelerador linear e qualidade de vida. Persp online: biol \& saúde. 2015;19(5):53-62. doi: https://doi. org/10.25242/88685192015885

19. Geinitz H, Zimmermann FB, Thamm R, et al. Fatigue in patients with adjuvant radiation therapy for breast cancer: Long-term follow-up. J Cancer Res Clin Oncol. 2004;130(6):327-33. doi: https://doi.org/10.1007/ s00432-003-0540-9

20. Lamino DA, Pimenta CAM, Braga PE, et al. Clinically relevant fatigue in women with breast cancer: prevalence and associated factors. Investig Enferm Imagen Desarr. 2015;17(2):157-168. doi: http://dx.doi.org/10.11144/ Javeriana.ie17-2.fcrm

21. Montgomery GH, David D, Kangas M, et al. Randomized controlled trial of a cognitive-behavioral therapy plus hypnosis intervention to control fatigue in patients undergoing radiotherapy for breast cancer. J Clin Oncol. 2014;32(6):557-63. doi: http://dx.doi. org/10.1200/JCO.2013.49.3437

22. Andrykowshi MA, Donovan KA, Laronga C, et al. Prevalence, Predictors, and Characteristics of OffTreatment Fatigue in Breast Cancer Survivors. Cancer. 2010;116(24):5740-5748. doi: https://doi.org/10.1002/ cncr. 25294 